\title{
Maquetas y modelos virtuales en el análisis constructivo básico de los edificios
}

\author{
Vicente López Mateu ${ }^{a}$
}

${ }^{a}$ Departamento de Construcciones Arquitectónicas ETSAV (UPV).viloma@csa.upv.es

\section{Resumen}

Los modelos y representaciones de construcciones han tenido un uso bastante extendido en distintos ámbitos humanos y disciplinas técnicas, especialmente útiles y populares en estos como instrumentos de trabajo para el análisis y representación de proyectos de edificación.

Esta situación ha evolucionado recientemente con la incorporación de otros medios y recursos informáticos que permiten resultados visuales similares, llegando recientemente a la realidad virtual o la confección directa de esas maquetas mediante impresoras tridimensionales.

El objeto de esta comunicación es presentar las recientes experiencias desarrolladas en la asignatura de Introducción a la Construcción de la E.T.S. de Arquitectura de Valencia, aportando aquellos aspectos más significativos para la reflexión sobre estas cuestiones en el ámbito docente.

La metodología utilizada ha sido la utilización de los medios tradicionales para elaborar maquetas aplicando un enfoque particular $y$ complementándolos con los nuevos medios o recursos disponibles, verificando las ventajas e inconvenientes y el progreso de los estudiantes.

Los resultados obtenidos permiten reconocer unos criterios generales sobre el uso más adecuado de estos recursos en el ámbito de la enseñanza de la arquitectura en etapas iniciales, en particular para el aprendizaje básico de los aspectos constructivos.

Palabras clave: modelos virtuales aplicados a la enseñanza, maquetas de arquitectura, diseño tridimensional conceptual. 


\title{
Virtual models in the basic constructive buildings analysis
}

\author{
Vicente López Mateu ${ }^{\mathrm{a}}$
}

${ }^{a}$ Departamento de Construcciones Arquitectónicas ETSAV (UPV). viloma@csa.upv.es

\begin{abstract}
The models and representations of buildings have had a fairly widespread use in various human areas and technical disciplines. They have been especially useful and popular as working tools for the analysis and representation of building projects.

This situation has changed recently due to the addition of computing resources which allow similar visual results. These working methodologies recently allow reaching virtual reality or direct preparation of these models using three-dimensional printers.

The purpose of this communication is to present the recent experiences developed in the subject Introduction a la Construccion from the E.T.S. Architecture in Valencia, providing a reflect on the most significant aspects for these issues in teaching.

The methodology used is a combination of the traditional way to develop models using a particular approach and complementing them with new resources available, verifying the advantages and disadvantages and student progress.

The results allow us to recognize general criteria on the most appropriate use of these resources in the field of architectural education in the early stages, particularly for basic learning of the constructive aspects.
\end{abstract}

Keywords: virtual models applied to construction analysis, three-dimensional design conceptual models 


\section{Introducción}

Desde la antigüedad las representaciones en maquetas de los edificios se han utilizado por la mayoría de las culturas con fines diversos, no solamente de tipo técnico sino representativo, religioso o incluso funerario (Azara, 1997). Aunque dado su carácter generalmente efímero se han conservado pocos ejemplares, gracias a ellas hemos podido conocer en algunos casos la imagen que nuestros antepasados tenían de su arquitectura.

Las maquetas eran, y siguen siendo, modelos en miniatura, utilizadas como puente de conexión entre las ideas y la realidad, entre lo abstracto y lo concreto. Con estos elementos los seres humanos en general, y particularmente los arquitectos, han conseguido visualizar de manera tangible el espacio arquitectónico y sus distintos elementos: los volúmenes, los vacíos, los colores y texturas, así como las relaciones entre ellos, es decir, las consideraciones básicas de todo proyecto arquitectónico.

El análisis y desarrollo de muchas obras complejas construidas en el pasado han requerido descripciones escritas y diferentes formas de representación gráfica, recogidas progresivamente en los planos y en algunos casos bastante conocidos, la elaboración de modelos específicos. Han sido numerosos los arquitectos, ingenieros y artistas que han utilizado esta técnica: desde Miguel Angel o Leonardo da Vinci a Gaudí, Le Corbusier, etc. Muchos de ellos han sido representados o fotografiados trabajando con sus maquetas.

Por lo tanto, estas maquetas no son únicamente elementos decorativos ni juguetes, sino una herramienta fundamental de trabajo aplicable en cualquier fase del proyecto, ya sea creativa, de reflexión o para el análisis, con distintas formas, presentación y desarrollo (Knoll y Hechinger, 2009). Su empleo progresivo ha venido motivado por la novedad y complejidad que suponían algunas construcciones que conjugaban distintos aspectos, así como por la necesidad de mostrarlas o explicarlas a distintas personas.

De este modo la realización de un proyecto y la toma de decisiones se han convertido en un proceso colectivo y la elaboración de un modelo generalmente es algo global, desde su idea y su concepción, a la confección en sí misma; llegando hasta la posterior exposición y recopilación de opiniones al respecto. Los edificios en el fondo son elementos tridimensionales, cada vez más complejos de proyectar y de construir, procesos en los que intervienen muchas personas, por lo que la suma de esfuerzos es un aspecto fundamental.

De manera similar, pero cambiando la escala a la de la ciudad, algo similar ocurre con los espacios donde se van a insertar estos objetos, o los que van a generar cuando se trata de una nueva urbanización. Resulta necesario, casi indispensable en ciertos casos, reconocer previamente los efectos de su inserción en un conjunto o en un paisaje, ya sea natural o urbano y sus posibles efectos y consecuencias, ya que van a conformar el hábitat humano durante un periodo largo de tiempo.

Por todos estos motivos, los modelos y maquetas han sido, y siguen siendo, una herramienta muy efectiva y adecuada para hacer comprensibles aspectos de la edificación y el urbanismo. Pero cabe preguntarse en qué sentido se pueden utilizar para mejorar la enseñanza de la arquitectura en general o en qué aspectos más concretos y particulares del aprendizaje puedan aplicarse.

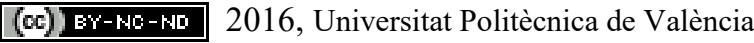

Congreso In-Red (2016) 


\section{Objetivos}

\subsection{Situación actual, comparación de los distintos ámbitos de aplicación}

El uso de las maquetas hoy en día es habitual, desde trabajos académicos, proyectos de graduación o de Máster o incluso concursos que pueden realizar los propios estudiantes. No obstante, los planteamientos están cambiando con la introducción de los nuevos recursos tecnológicos y de transmisión de la información que generan nuevas posibilidades de trabajo y visualización.

El uso de maquetas en ámbitos formativos y profesionales es un hecho bastante común y con cierta tradición, aunque su extensión y uso es diferente en áreas como la Ingeniería de Caminos, Ingeniería Industrial o campos más específicos como la el diseño de mobiliario o la aeronáutica. Las escalas, el nivel de detalle y los aspectos técnicos así como los materiales utilizados suelen ser diferentes, aunque los propósitos generales son similares.

Por otro lado, el uso de maquetas dentro de la formación en Arquitectura y Arquitectura Técnica o Ingeniería de la Edificación, es común y con amplia tradición en las asignaturas como Proyectos o Estructuras. En estas asignaturas, frente a las que se desarrollan en construcción, prevalecen otras características como el aspecto final de los edificios, los volúmenes, el espacio que generan o la disposición de su estructura.

En el ámbito académico, la aplicación de las maquetas arquitectónicas requiere distinguir dos aspectos: el planteamiento general y los materiales o medios utilizados para su confección. Actualmente resulta sencillo encontrar distintas publicaciones y recursos que abordan el uso de distintas técnicas, materiales y procesos para su elaboración (Navarro Lizandra, 2005; Knoll y Hechinger, 2009), pero en pocos casos se trata de manera exhaustiva los planteamientos o fines propios que subyacen tras la realización de estos modelos (Consalez, 2006).

Estas técnicas y procesos resultan fundamentales para conseguir un acabado más o menos figurativo o representativo de la realidad que se pretende representar, aunque en algunas asignaturas, desde el punto de vista académico, este puede no ser el aspecto más relevante. Por ello resulta necesario incidir en los planteamientos y objetivos para su utilización, así como en aspectos concretos como el plazo en el que se deben concebir y realizar los modelos.

\subsection{Aspectos materiales y tecnológicos, la evolución y aplicación de técnicas y recursos}

Habitualmente se han utilizado maquetas confeccionadas con distintos materiales tradicionales: cartón, madera, escayola, corcho, etc., y más recientemente con materiales compuestos o sintéticos: cartón pluma, plexiglás, poliestireno, etc. o la combinación de todos ellos. Aunque su manipulación o ensamblaje resulta más compleja utilizando los medios artesanales, también se han incorporado pequeñas piezas o elementos metálicos y recientemente otros materiales reutilizados o reciclados.

En arquitectura, a diferencia de otros usos artísticos o industriales de tipo general, se utilizan poco los moldes, ya que no resulta frecuente realizar la repetición de una maqueta. En muchos casos se trata de un modelo único similar a una escultura, con fines de proyecto más o menos concluido, o bien de análisis parcial, conocidas como "maquetas de trabajo".

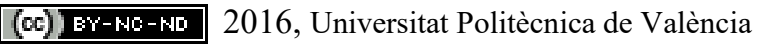


En esta situación los materiales utilizados en la confección de las maquetas pueden considerarse ya un aspecto expresivo y representativo en sí mismo, relacionado, en su caso, con su construcción posterior.

Pero dejando a un lado esta singularidad, cabe plantearse de qué modo se genera y se transmite la información actualmente. En este sentido, se puede reconocer que la información sobre arquitectura en la Web está basada en los siguientes componentes:

- Textos alfanuméricos en distintos idiomas o alfabetos.

- Dibujos o imágenes y sus combinaciones.

- Fotografías de elementos reales o virtuales.

- Sonidos: voz humana, música, maquinaria, etc.

- Videos: imágenes reales, animaciones o combinaciones de ambas.

Las maquetas tradicionales de arquitectura utilizan principalmente los tres primeros, frente a los modelos virtuales que pueden utilizar todos ellos. Por lo tanto, las posibilidades de manejo y transmisión de información aumentan con los programas informáticos que facilitan su gestión. Por este motivo el desarrollo del diseño asistido por ordenador conocido por CAD (Computer-Aided Design) y los programas de representación tridimensional han experimentado un gran progreso en los últimos años, permitiendo modelos cada vez más completos y próximos a la realidad.

Siguiendo los pasos de este desarrollo, cada vez han ido introduciéndose con más fuerza los programas o aplicaciones informáticas BIM (Building Information Modelling), que tienen la virtud de almacenar o relacionar en un mismo archivo informático toda esta información. Ofrecen generalmente representaciones tridimensionales de los edificios que superan las infografías clásicas, ya que permiten combinar múltiples y diversos aspectos de forma completa y compleja.

De este modo se ha llegado a enlazar distinta información gráfica con la información alfanumérica, materiales, acabados, bases de precios, etc. haciendo posible integrar y desarrollar simultáneamente aspectos muy específicos o concretos de los edificios. De este modo, se combinan las cuestiones o aspectos generales, con otros datos de tipo técnico y constructivo, que permiten un proceso de proyecto o análisis más completo y próximo a la realidad.

Cabría pensar que todo esto desplazaría a la realización de maquetas o modelos materiales; sin embargo, y apoyados en este mismo desarrollo, las tendencias más recientes refuerzan la utilización de maquetas empleando nuevas tecnologías. Las denominadas "impresoras 3D", cada vez más rápidas y menos costosas, permiten fabricar directamente objetos tridimensionales por superposición de finas capas de materia fluida que posteriormente se solidifica.

Esto plantea ciertas ventajas respecto a los métodos más o menos artesanales precedentes, especialmente en cuanto a la precisión y rapidez, así como a la posible repetición de los modelos, aunque todavía se encuentran limitadas en cuanto al tamaño de las piezas. Durante 
algún tiempo esta tecnología de diseño y fabricación ha sido utilizada casi exclusivamente por empresas pioneras, pero en los últimos años su uso se ha popularizado notablemente.

Se constata fácilmente que la impresión 3D está en auge y las perspectivas sugieren en el futuro próximo un gran impacto en la sociedad. Sus defensores lo llegan a relacionar con el ahorro y el desarrollo sostenible, hablándose de los "nuevos creadores", los "artesanos del 3D digital". Según Biehler y Fane (2014) estos profesionales individuales y sus microempresas sustituirán progresivamente la cultura del consumo y del despilfarro actual por una cultura de la creación, la innovación y la producción descentralizada.

Todas estas herramientas se han ido desarrollando e influenciándose simultáneamente, y en estos momentos resultan factores claves para su elección e implantación en determinadas tareas por la facilidad de uso y su compatibilidad, relacionada esta última con el intercambio sencillo de información. En cualquier caso, cabe mencionar ciertas barreras y limitaciones como los equipos informáticos, humanos y de software especializados así como la inversión inicial económica y de tiempo necesario para su dominio.

Por este motivo, despierta también el interés desde el punto de vista pragmático y pedagógico, trabajar en el mundo de la configuración tridimensional de la arquitectura introduciéndose progresivamente hacia estas tecnologías, pero sin dejar la utilización de materiales o soluciones que podríamos denominar de "baja tecnología" (Navarro Lizandra, 2005). Esto permitiría soluciones rápidas a bajo coste y de tipo más bien conceptual, que harían posible una rápida comunicación con poca inversión de recursos, lo que suele precisarse en los ámbitos académicos actuales.

\subsection{Planteamiento adaptado a la docencia en construcción}

Siguiendo la amplia tradición, y atendiendo a las recientes innovaciones en el campo de la arquitectura, la realización de modelos y las representaciones gráficas de distinto tipo se utilizan como elemento habitual de aproximación a la realidad física y material de las construcciones. La representación de la arquitectura tiene como objeto generalmente adelantar el resultado de la obra proyectada.

Esto se produce en diferentes fases o estadios: el esquema conceptual, el boceto o el dibujo del croquis y finalmente la representación a escala del proyecto, bien sea en dos o tres dimensiones. Sin embargo, en este caso se pretende aplicar particularmente al reconocimiento de los sistemas constructivos y de los principales elementos que los componen. Se trata de un procedimiento necesario y complementario de otros, como su descripción mediante textos, fotografías, o más recientemente vídeos o animaciones sobre procesos constructivos.

Por lo tanto, se plantea la necesidad de incidir por un lado en los aspectos más conceptuales de una construcción y, por otro lado, en sus detalles particulares que en muchas ocasiones se encuentran ocultos o no resultan evidentes cuando se ha concluido la fase de construcción. En este sentido resultan más útiles los modelos "interpretativos" frente a los "representativos" de la realidad, en los que pueden, y deben, ser consideradas cuestiones diferentes. Por lo tanto, se deben dejar de lado aspectos más "expresivos", procurando intencionadamente conseguir otros más técnicos o "conceptuales".

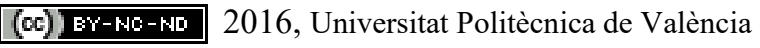


Las maquetas en las asignaturas de Construcción pueden ser similares, pero no idénticas a las utilizadas en otras disciplinas, aunque deben tener otro planteamiento más relacionado con aspectos generales, basado en cómo se conforma o materializa un edificio, incluso como se construye paso a paso, elemento a elemento. Esto resulta clave hoy en día, por la dificultad de visitar obras por los estudiantes, teniendo en cuenta las condiciones de seguridad que requieren estas visitas, que en todo caso serían parciales.

Siguiendo la metodología habitual y bastante consolidada del diseño de modelos y maquetas con distintos materiales (Navarro Lizandra, 2005), cuando un proyectista se enfrenta a un nuevo trabajo se establecen varias fases entre las que cabe destacar:

1. Representación de la idea por medio de dibujos (croquis, apuntes o bocetos), produciendo una aproximación progresiva a la propuesta o solución planteada.

2. Construcción de una o varias maquetas sencillas, mediante materiales simples, que permiten obtener o verificar la idea y los cambios o modificaciones iniciales.

3. Realización de prototipos que pueden experimentarse a escala real de manera formal y en algún caso funcional.

En lo que respecta a la arquitectura, en pocas ocasiones se alcanza el último paso, aunque pueden realizarse "proyectos piloto" o edificios experimentales cuando se propone la construcción de los edificios o complejos urbanos por suma o repetición de elementos. En el caso de análisis de construcciones y estudio de los edificios a nivel académico, por lo general el proceso se detiene en la segunda fase, denominándose "maquetas de trabajo".

En este caso se trata de utilizar ese aspecto preciso, las maquetas que pueden realizarse de manera sencilla y rápida para realizar un análisis preliminar a la vez que reflexivo sobre las características constructivas de un edificio determinado. Se trata así de no realizar un modelo o un trabajo concluido, sino que pueda utilizarse de manera rápida como una herramienta adicional o complementaria del proceso de análisis.

De manera similar, aunque no podemos establecer en este caso que exista la misma metodología, cabría utilizar los programas informáticos que permiten la realización de modelos o "maquetas virtuales" en tres dimensiones. En este segundo caso se podrían establecer ciertas dudas a la vez que ciertas ventajas, ya que el modelo estaría entre el primer y el segundo paso mencionados. En esta situación se pueden producir mayor número de modelos a partir de la idea inicial o iteraciones entre el modelo y los bocetos iniciales.

El primer paso se realiza a mano alzada, sobre un soporte físico de papel, un ordenador portátil o sobre una tableta informática. En el segundo paso, se abandonan ya los utensilios clásicos de dibujo y se realiza una maqueta con materiales sencillos o se emplean recursos informáticos. El objetivo final de esta segunda parte será obtener un modelo de trabajo, que comprende los planos de análisis $2 \mathrm{D}$, y las representaciones 3D de la forma arquitectónica, maquetas o representaciones icnográficas.

\section{Desarrollo de la innovación}

\subsection{Planificación general}

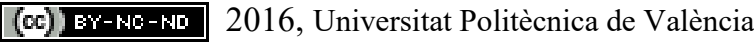

Congreso In-Red (2016) 
Dentro de los aspectos fundamentales asignatura, cabe destacar que "Introducción a la Construcción" es una asignatura troncal que pertenece al primer curso y primer cuatrimestre de estudios del Grado en Fundamentos en Arquitectura. Por lo tanto, se trata de abordar la construcción y la elaboración de modelos arquitectónicos de forma elemental, sin conocimientos previos y con técnicas sencillas.

Por otro lado, como ya se ha expuesto anteriormente, siguiendo las posibilidades de participación y aprendizaje colectivo que permite la realización de estas maquetas, cabe implementar otros objetivos de tipo transversal. Estos objetivos y competencias deben plantearse y formularse de forma separada, distinguiendo los relacionados con la propia asignatura de los de tipo general, aunque ambos deben ser evaluados y considerados.

De este modo, entre los primeros objetivos, los relativos a la asignatura, cabría destacar:

- Reconocer y visualizar el espacio arquitectónico

- Explorar la complejidad constructiva de la edificación

- Identificar los distintos elementos de un sistema constructivo

- Interpretar planos y representaciones técnicas específicas de los edificios

- Comparar los distintos sistemas constructivos y sus características principales

De manera simultánea a este planteamiento se pretende alcanzar el aprendizaje y la evolución en otras competencias generales, instrumentales y transversales básicas, que se consideran fundamentales para los alumnos de los primeros cursos de la formación universitaria. En este caso, se utiliza el propio modelo, su proceso de elaboración y posterior exposición pública como base de estos aprendizajes.

Respecto a los objetivos relacionados con las competencias transversales, cabe citar:

- Aprendizaje autónomo y colectivo de los estudiantes

- Capacidad de observación, deducción e interpretación

- Organización y planificación del trabajo individual y en equipo

- Comunicación activa, exposición y defensa pública de un trabajo

Según la experiencia acumulada en los últimos años, estos objetivos están relacionados, y generalmente la consecución de objetivos relativos a competencias transversales refuerza notablemente los específicos. En este sentido, es fundamental que los grupos de trabajo realicen las distintas tareas de forma coordinada y cohesionada, que sus componentes adquieran un alto grado de implicación y motivación por el trabajo común.

La combinación adecuada entre estos objetivos y su equilibrio es un aspecto fundamental para que esta metodología se aplique adecuadamente. Esto no impide que se alcancen también otros objetivos más particulares, relativos a cada una de las unidades o sistemas específicos a reconocer dentro de un edificio: estructura, particiones, envolvente, etc.

\subsection{Adaptación y organización particular a la asignatura}

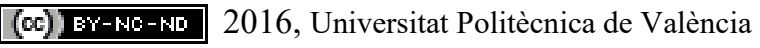


En la asignatura de "Introducción a la Construcción" de primer curso los alumnos han desarrollado en los últimos 10 años distintos tipos de trabajos en equipo utilizando de manera complementaria los medios expuestos: las maquetas y modelos virtuales, bien de manera simultánea, sucesiva o alternativa. Estos trabajos de tipo práctico forman parte del aprendizaje teórico-práctico que se ha desarrollado en las actividades prácticas.

La aplicación de las maquetas en el aprendizaje se utiliza actualmente en dos trabajos. El primero de ellos está relacionado con el análisis de un edificio modelo o ejemplo y en el segundo con el estudio del comportamiento de las estructuras de barras trianguladas (LopezMateu, 2013). En ambos casos los alumnos realizan un modelo tridimensional utilizando elementos sencillos, concluyendo en ambos casos con la exposición del modelo y de las conclusiones del análisis o de la propuesta.

Estos dos ejercicios se realizan a principio de curso, aunque su duración ha ido modificándose en función de la planificación anual de la asignatura y de los créditos asignados a la misma en las recientes modificaciones del Plan de Estudios y del Grado en Fundamentos de arquitectura. Poco a poco se ha centrado el mayor interés en el primero que se desarrolla durante todo el cuatrimestre, siendo utilizado para la práctica de los aspectos concretos de cada unidad sobre el mismo edificio.
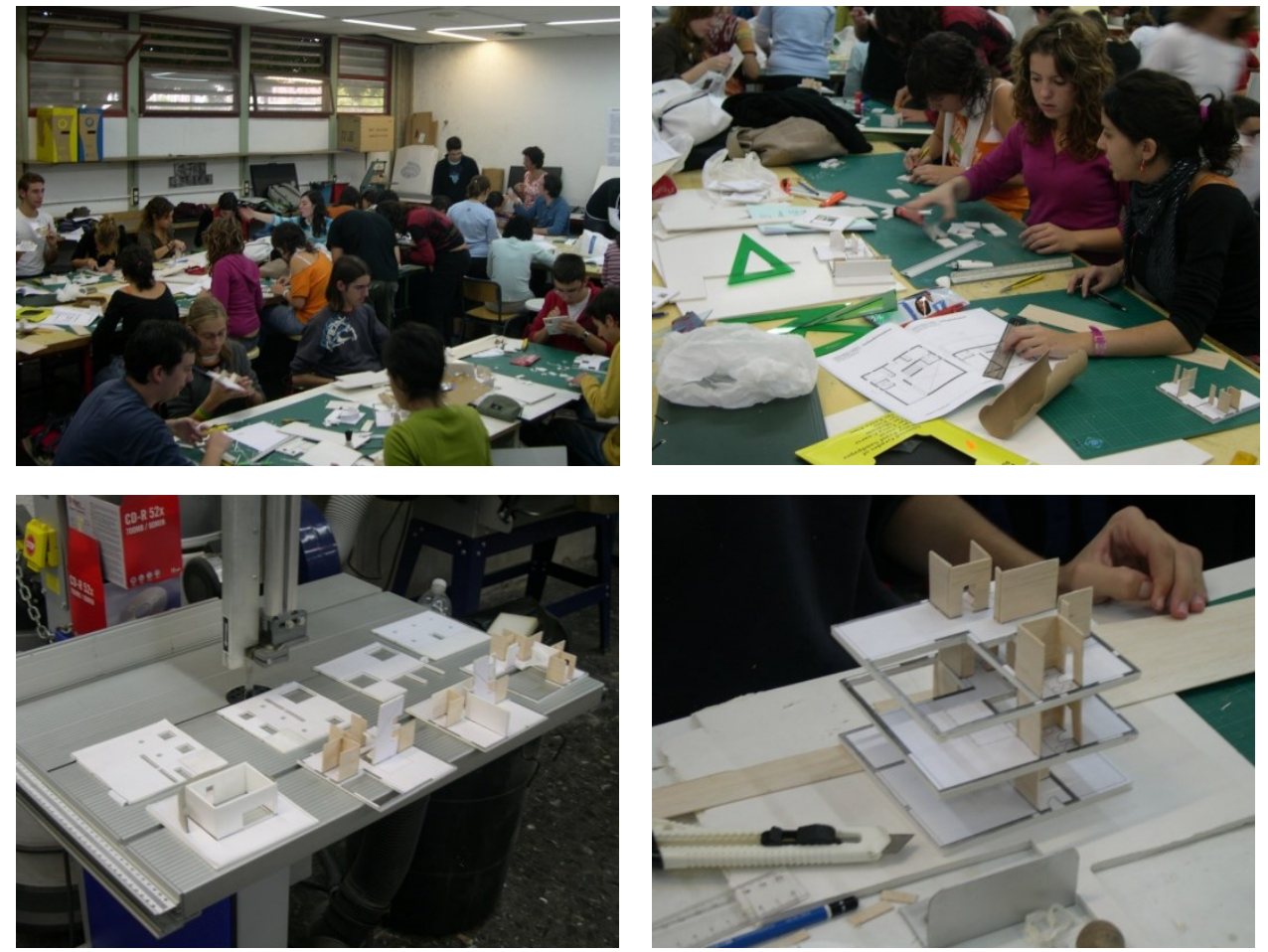

Fig. .1 Secuencia de imágenes de los estudiantes trabajando en la elaboración de maquetas Trabajo del curso 2005-06 de los Grupos C, E y G.

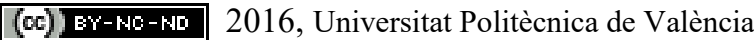

Congreso In-Red (2016) 

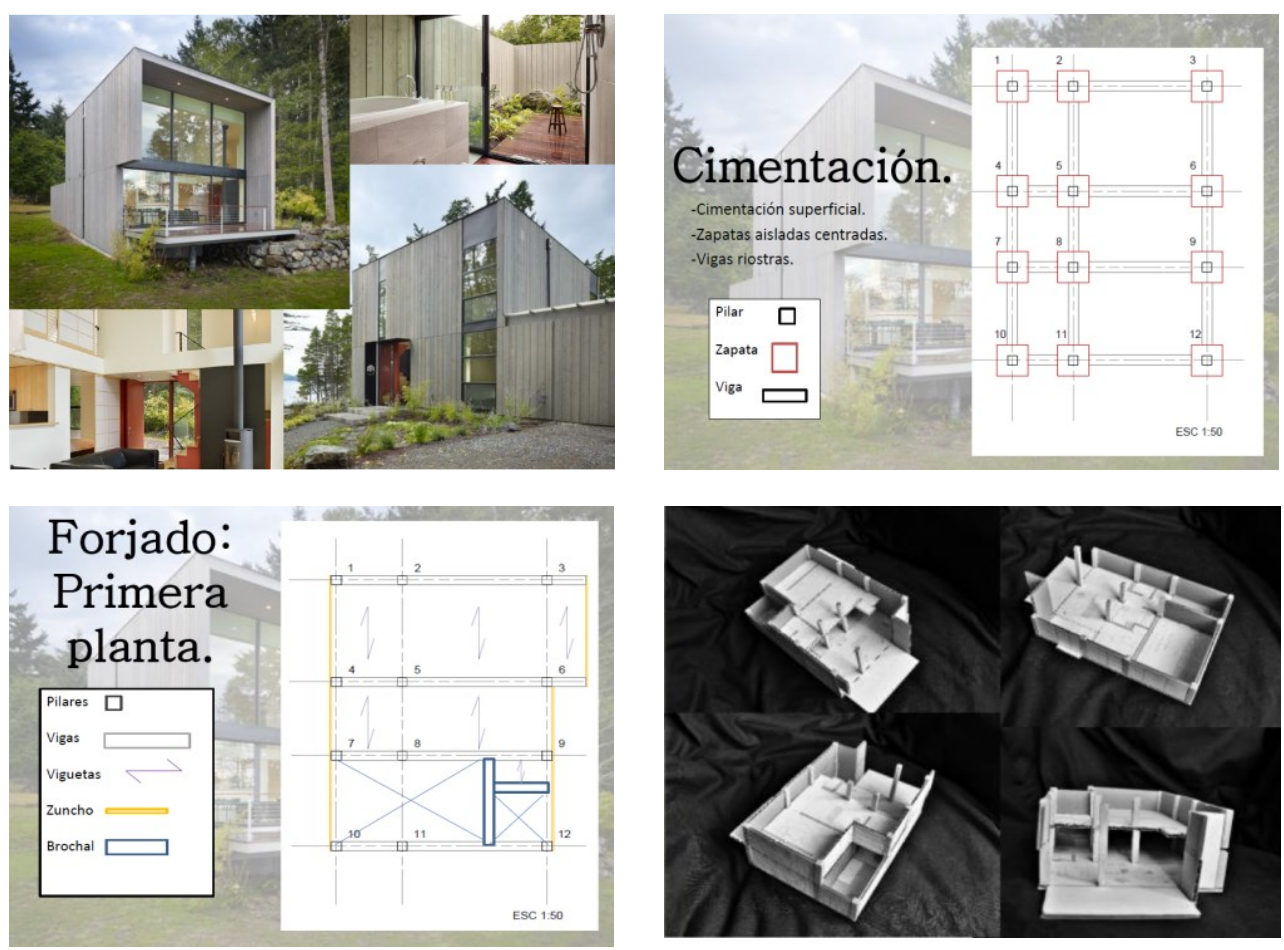

Fig. 2. Análisis de una vivienda: cimentación, estructura y maqueta Trabajo del curso 2015-16 realizado por C. Gutiérrez, V. Pérez, C. Di Lorenzo, M. A. Muntaner y P. Martínez.

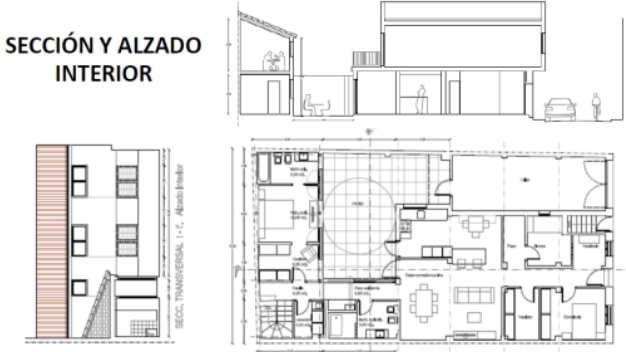

CARACTERÍSTICAS DISTRIBUTIVAS GENERALES

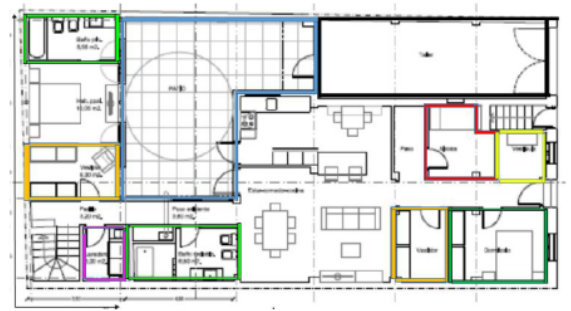

IBUCIÓN DE LA VIVIENDA

COMUNICACIÓN VERTICAL
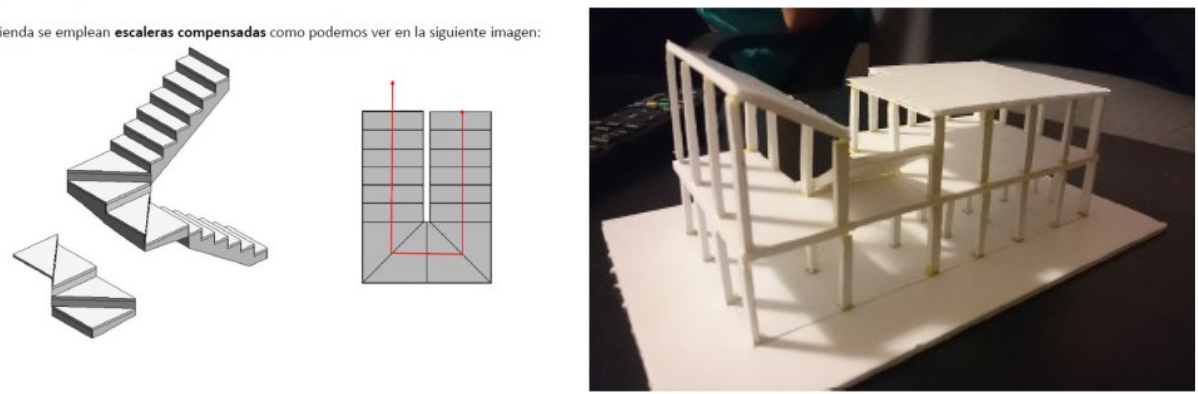

Fig. 3. Análisis general de una vivienda, espacios, escalera y estructura. Trabajo del curso 2015-16 realizado por M. Gómez, D. Muñoz, D. Munuer y M. Fuster.

(cc)) EY-NC-ND 2016, Universitat Politècnica de València

Congreso IN-RED (2016) 

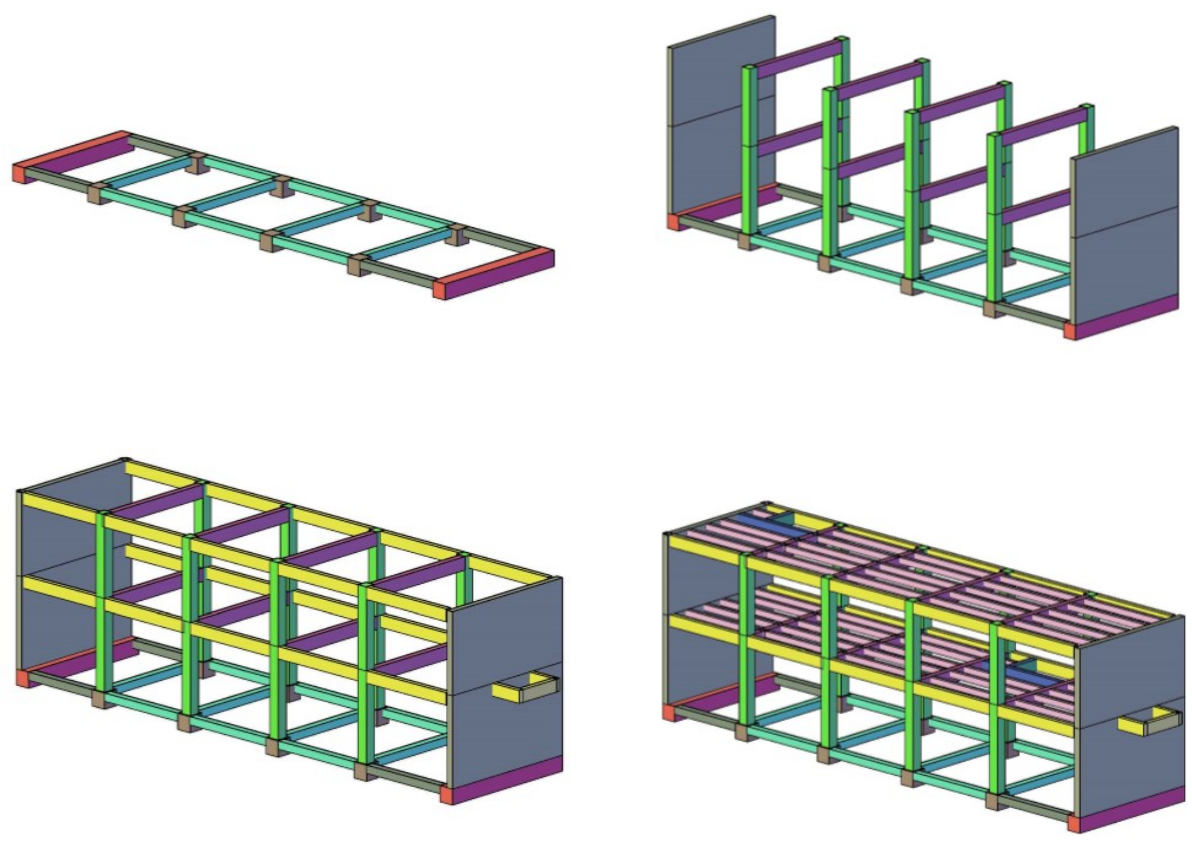

Fig. 4. Análisis de la estructura de un edificio: cimentación, pilares, vigas y forjados. Trabajo del curso 2015-16 realizado por F. Fabuel V. Fortuny, J. García, E. Rodríguez y L. Valls.
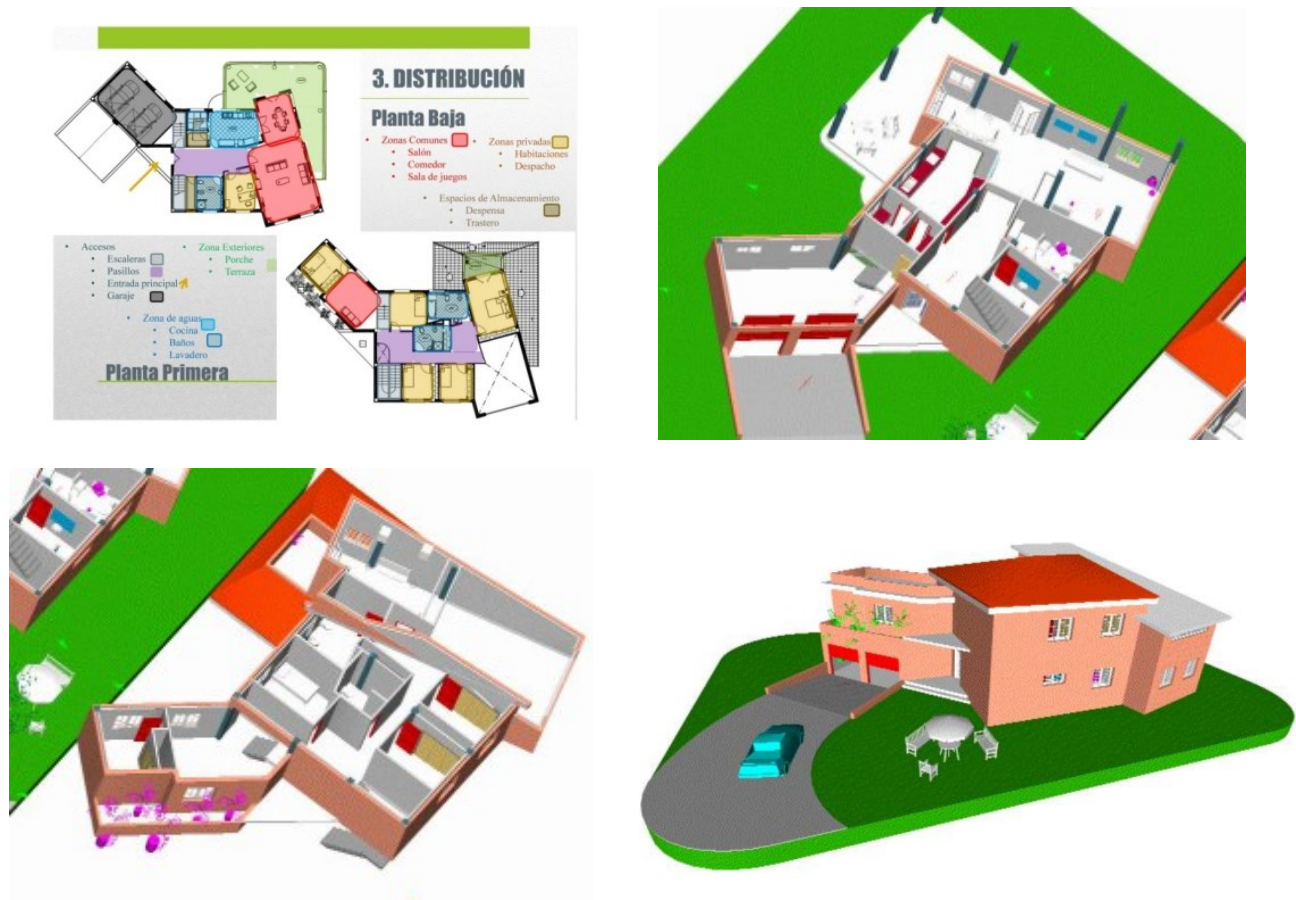

Fig. 5. Análisis de la distribución de una vivienda, su estructura, particiones y envolvente. Trabajo del curso 2015-16 realizado por A.M. Izquierdo, J. C. Chueca J. Redón, I. Colomer y J. Edo.

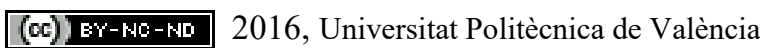

Congreso In-Red (2016) 


\subsection{Desarrollo de la actividad}

El planteamiento de los dos casos resulta totalmente diferente. En el primer caso se trata de reconocer la representación de la arquitectura, generalmente en planos técnicos más o menos complejos y entender aquellos elementos o detalles particulares. En este por lo general se utilizan unos sistemas de representación y unos códigos o convenciones que resultan complejas para los estudiantes (Pérez Carrión, 2006).

Los edificios se representan mediante planos y maquetas sencillas elaboradas con materiales como el cartón o el papel, económicos y fácilmente reciclables. En el caso de modelos virtuales suelen presentarse acompañados de textos y en algunos casos de videos. Esto último enriquece los resultados aunque no debe desviar la atención de los estudiantes de los aspectos fundamentales del análisis de los edificios. Los aspectos más avanzados pueden introducirse en etapas posteriores de formación de los alumnos.

Debido a la gran evolución que la tecnología del diseño asistido por ordenador ha tenido en los últimos años y los avances en los programas de modelado, los estudiantes pueden encontrar una gran variedad y diversidad de programas, muchos de ellos gratuitos, con los que realizar estos modelos. Por otro lado, la introducción en este campo de las técnicas digitales resulta más que necesaria.

\subsection{Evaluación}

El conocimiento alcanzado por los estudiantes se determina sobre la presentación y memoria elaborada, así como sobre las explicaciones realizadas sobre la propia maqueta, que pasa a convertirse en la herramienta más importante en el proceso de aprendizaje. Este segundo paso se evalúa con una rúbrica, valorándose aspectos como la propia presentación y explicación, en el que participan sus compañeros.

La evaluación parcial y final se realiza a través de la presentación de estos trabajos en la plataforma PoliformaT de la UPV. Los trabajos reciben la calificación y los comentarios del profesor, permitiendo de este modo la retroalimentación y en su caso la mejora de los trabajos académicos que cada grupo presenta. La nota final engloba todos los apartados del análisis y los aspectos valorados.

Los alumnos disponen de plantillas y herramientas de trabajo colaborativo, sistemas de compartir e intercambiar de archivos y de comunicación entre los componentes del grupo de tipo chat y foros. Estas últimas herramientas suelen utilizarse más para la organización interna de los grupos que para el desarrollo del trabajo en esta tarea concreta, dando preferencia entre ellos al trabajo en el aula.

\section{Resultados}

\subsection{Recopilación de las opiniones de los alumnos y rendimiento académico}

Ante la dificultad de obtener y comparar los resultados de una actividad tan compleja y evaluar el aprendizaje efectivo que se produce, así como su aceptación por parte de los estudiantes, se recurre a uno de los sistemas clásicos utilizados en la evaluación del aprendizaje, las respuestas escritas de los propios estudiantes (Schunk, 1997).

(c)) EY-NC-ND 2016, Universitat Politècnica de València 
En este sentido, cuando se han realizado varios tipos de prácticas, aproximadamente en el periodo intermedio de formación se realiza una "encuesta abierta" a los alumnos en la que se les pide que evalúen por escrito los aspectos positivos y negativos generales y particulares de la asignatura. Esto resulta difícil de cuantificar a partir de las encuestas generales o de opción múltiple, por lo que se recogen sus opiniones con este formato.

En el caso de las prácticas, esta actividad de la confección de maquetas es la que recibe una valoración más positiva por parte de los alumnos frente a otras prácticas de la propia asignatura. En las encuestas realizadas la valoración positiva oscila entre los porcentajes del $70-80 \%$ según los grupos encuestados. Por otro lado, la mayoría los estudiantes (92\%) suelen reclamar un periodo más dilatado para realizar estos modelos o espacios y medios específicos.

Entre los datos analizados de los resultados académicos destacan las mejores calificaciones de aquellos alumnos que utilizaron ambas técnicas, las maquetas y las representaciones virtuales (28\%), en segundo lugar aquellos que utilizaron las maquetas convencionales $(53 \%)$ y finalmente los que únicamente utilizaron los medios virtuales (19\%). En este último caso suele ser un estudiante el que realiza el modelo con ayudas puntuales.

\subsection{Aspectos positivos y negativos observados}

La representación de las ideas creativas resulta primordial en cualquier disciplina del diseño, y se manifiesta especialmente relevante en arquitectura, debiéndose entender también todo el proceso de análisis y síntesis que se produce en su confección. De este modo, las maquetas y modelos pueden ser objetos muy versátiles en estos procesos, pues permiten a los futuros arquitectos reflexionar y expresar sus pensamientos rápidamente, de un modo muy efectivo.

Dado que se trata de alumnos de primer curso sobre los que se realizado la experiencia, se observa que la actividad favorece notablemente la relación entre los componentes del grupo y la cohesión en general de los equipos de trabajo. Conseguir esto de manera rápida resulta fundamental en periodos iniciales de formación, dado el reducido periodo de formación que supone un cuatrimestre.

Este efecto es mayor en el caso de maquetas realizadas con medios y recursos convencionales frente a las maquetas virtuales, en las que el planteamiento debe evitar las tendencias al individualismo. Su propia ejecución debe permitir la participación de los miembros de los equipos en distintas tareas, que deben ser asignadas y revisadas puntualmente por los profesores en su labor de seguimiento y tutorización del trabajo.

Este aspecto se basa fundamentalmente en el desarrollo de habilidades comunicativas interpersonales relacionadas con el trabajo en equipo que se deben potenciar y fomentar (Rees, 1991). Los estudiantes pueden compartir y debatir de manera directa determinados aspectos de la tarea y el proyecto aprovechando su interacción sobre un modelo real y tangible. Esto es posible también en los modelos virtuales, pero resulta más complejo y requiere el empleo de programas comerciales o recursos específicos que permitan el trabajo colaborativo en "tiempo real".

(c)) EY-NC-ND 2016, Universitat Politècnica de València

Congreso In-Red (2016) 
Posteriormente, para la redacción de los documentos de la memoria, la presentación o exposición final del trabajo, se ha comprobado que resulta positivo el empleo de algunas herramientas donde los estudiantes pueden trabajar simultáneamente proporcionadas por distintas plataformas como Google Drive, o el trabajo en plataformas colaborativas. De esta forma los profesores también pueden comprobar las distintas aportaciones en el trabajo conjunto.

Deben evitarse ciertos errores recurrentes en la concepción y ejecución de estos elementos buscando la mayor simplicidad y abstracción en los resultados, frente a otros aspectos como la apariencia o la visualización. Asimismo resulta necesario salvar ciertas dificultades de su realización por desconocimiento de los materiales o elementos seleccionados, así como de los programas utilizados en el caso de que se realicen mediante una aplicación informática.

El estudio de edificios aportados por los alumnos genera una gran dispersión, casos particulares que por una parte enriquecen las posibilidades pero por otra parte generan una gran dispersión y soluciones en algunos casos un tanto atípicas o propias de las circunstancias del entorno o de la distribución del edificio. Es preferible que los ejemplos a estudiar sean facilitados previamente por los profesores, que deben estudiarlos previamente.

\section{Conclusiones}

Las maquetas y modelos tridimensionales sencillos utilizados en el aprendizaje inicial de la construcción arquitectónica se muestran como una herramienta muy útil y versátil que permite introducir fácilmente al alumno en los procesos de estudio y comprensión tridimensional de los edificios.

Frente a los planteamientos de otras asignaturas o campos basados en la imagen o la apariencia de los edificios, para los estudiantes resulta muy positivo reconocer la compleja realidad constructiva de los edificios a través de modelos. Desde periodos iniciales resulta imprescindible que analicen e interpreten esta cuestión, reforzándola con relaciones directas y sensoriales como la realización de este tipo de prácticas.

Esta herramienta requiere su adaptación académica a las condiciones de aprendizaje inicial de construcción. En este ámbito existen varias particularidades propias y diferenciadas de otros usos más extendidos y comunes hasta ahora. Para ello es necesario concentrar los esfuerzos en los aspectos conceptuales, por encima de los expresivos o figurativos. Sobre esta base se pueden ir añadiendo estos y otros aspectos en posteriores etapas formativas.

Los estudiantes valoran mayoritariamente de forma muy positiva la realización de esta actividad, siendo la preferida respecto a otro tipo de actividades, reforzando las habilidades de trabajo en equipo en base a una comunicación directa y efectiva, así como el propio reconocimiento temprano de las capacidades y los roles de los miembros del equipo.

Por lo tanto, debe potenciarse el uso de maquetas y modelos en los ámbitos académicos desde el inicio del aprendizaje, considerando que su importancia no sólo como ayuda en el crucial proceso de diseño y de toma de decisiones, sino también como medio para generar, investigar y desarrollar en equipo aspectos de análisis y creativos como complemento de los nuevos recursos y medios tecnológicos.

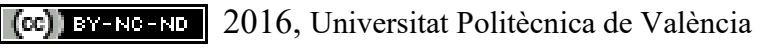




\section{Referencias}

AZARA, P. (1997). Las casas del alma: maquetas arquitectónicas de la antigüedad, 5500 a.C-300 d.C. Barcelona: Fundación Caja de Arquitectos.

BERCHON, M. y LUYT B. (2016). La impresión 3D. Barcelona: Gustavo Gili.

BIEHLER J. y FANE, B. (2014) Impresión 3D con Autodesk. Crear e imprimir objetos 3D con 123D, Autocad e Inventor. Madrid: Anaya Multimedia, S.A.

BOSWORTH, K. (1994). Developing Collaborative Skills in College Students. New Directions for Teaching and Learning, 59. San Francisco: Jossey-Bass. pp. 25-31.

CONSALEZ, L. (2006). Maquetas: la representación del espacio en el proyecto arquitectónico. Barcelona: Gustavo Gili.

DUNN, N. (2010). Maquetas de arquitectura. Medios, tipos, aplicación. Barcelona: Blume (Naturart S.A.).

DUNN, N. (2012). Proyecto y construcción digital en arquitectura. Barcelona: Blume.

KNOLL, W. y HECHINGER, M. (2009). Maquetas de arquitectura.: Técnicas y construcción. Barcelona: Gustavo Gili.

LÓPEZ MATEU, V. y BENLLOCH MARCO, J. (2013) "Diseño y elaboración de modelos estructurales para el aprendizaje inicial del comportamiento de estructuras en edificación” ACHE. En III Jornadas Internacionales de Enseñanza de la Ingeniería Estructural (Valencia 12 y 13 de junio de 2013). Valencia: Universitat Politècnica de València. 251-262.

NAVARRO LIZANDRA, J. L. (2005). Maquetas, modelos y moldes: materiales y técnicas para dar forma a las ideas. Colecció treballs d'Informàtica i tecnologia num. 4. Castellón: Universitat Jaume I.

PÉREZ CARRIÓN, T. et al. (2006). "Las maquetas como material didáctico para la enseñanza y aprendizaje de la lectura e interpretación de planos en la ingeniería”. INGEGRAF. En XVIII Congreso Internacional de Ingeniería Gráfica (Sitges 31 mayo, 1 y 2 junio de 2006). Barcelona: Universitat Politècnica de Catalunya. Disponible en <http:// http://rua.ua.es/dspace/handle/10045/21685> [Consulta: 30 de marzo de 2016]

REES, F. (1991) How to lead work teams: facilitation skills. San Francisco : Jossey-Bass/Pfeiffer.

SUCCAR, B. (2009). "Building information modelling framework: A research and delivery foundation for industry stakeholders". Automation in Construction. Vol 18 (2009) pp. 357-375. $<$ http://www.sciencedirect.com/science/article/pii/S0926580508001568> [Consulta: 15 de mayo de 2016]

SCHUNK, D. H. (1997). Teorías del aprendizaje. México: Pearson educación. Traducción de la obra Learning theories, an educational perspective (1996).

(c)) EY-NC-ND 2016, Universitat Politècnica de València

Congreso In-Red (2016) 